\title{
The Influence of Die Geometry on Stress Distribution by Experimental and FEM Simulation on Electrolytic Copper Wiredrawing
}

\author{
Leonardo Kyo Kabayama, Simone Pereira Taguchi, \\ Gustavo Aristides Santana Martínez* \\ Department of Materials Engineering - DEMAR, Engineering School of Lorena - EEL, \\ Universidade de São Paulo - USP, Pólo Urbo Industrial, \\ Gleba AI-06, PO BOX 116, 12600-970 Lorena - SP, Brazil
}

Received: October 21, 2008; Revised: August 27, 2009

\begin{abstract}
The study of die geometry is vital in determining the surface and mechanical properties of drawn wires, and consequently, their application. In this work, annealed electrolytic copper wire (ETP), with 0.5 mm original diameter was reduced by $19 \%$ in dies with $2 \beta=10^{\circ}$ and $18^{\circ}$ and $\mathrm{Hc}=35$ and $50 \%$. The best experimental results were then studied by the Finite Element Method to simulate residual stress distribution. The experimental results show that the friction coefficient decreases as the wire drawing speed increases, and that low $2 \beta$ and $\mathrm{Hc}$ values bring about the most favorable wiredrawing conditions. The simulation shows a variation in the axial and radial tensions, both for the compression and traction stresses on all regions during the wire drawing process. In conclusion, the influence of the internal die geometry on the drawn wire is clarified.
\end{abstract}

Keywords: tribology, finite element method, wiredrawing, die geometry

\section{Introduction}

The drawing process is used for the production of bars, wires and tubes, in which good quality surface and mechanical properties of the final products comply with specifications. Consequently, it is necessary to understand the behavior of the material during the process, which depends on several factors, such as die geometry, initial material properties, lubricant, drawing speed and the interaction of all these factors to reach the ideal process condition.

A forecast of the wire mechanical properties is obtained through the Finite Element Method (FEM) numerical analysis of the variation of wire stress curves during the wiredrawing process. The main advantage of the FEM is the capability of obtaining detailed information about the deformation mechanisms such as velocity, shapes, strains, stress curves, temperatures, or distribution of contact pressure. Some disadvantages, however, are the necessity of intense familiarity with the software program used and the investment in both: hardware and software ${ }^{1}$.

\subsection{Wiredrawing process}

The wiredrawing is a plastic metal forming process, generally performed at cold working conditions, in which a suitably clean and lubricant coated wire is pulled through a die, which is a rigid tool with wear resistant surface. The longitudinal section of the die reduction region is shown in Figure 1.

This process is a complex interaction of many parameters ${ }^{1,2}$, defined by:

- Wire properties (yield stress, elastic modulus, strain rate, strain hardening);

- Lubricant (friction coefficient, viscosity, surface treatment); and
- Die geometry (reduction angle, bearing region length, reduction area, and material).

The above mentioned parameters control the material deformation during the wiredrawing process, and consequently, the stress distribution through the cross section of the drawn wire. With the correct parameter control, increased productivity and die life expectancy, as well as the wire breaking reduction will take place throughout the process. The parameter control also defines the wire properties such as good torsion resistance, tensile strength and rupture resistance ${ }^{3}$.

Different zones through the hole, as shown in Figure 2, constitute the die inside geometry: entrance zone (I), reduction zone (II), bearing zone (III) and exit zone (IV). The internal die geometry and mainly the reduction zone constitute the most important condition ${ }^{4}$, for it is where the working angle $(2 \beta)$ is defined. When the compression stress is applied, the friction must be minimized, thus reducing the die wear. The friction between the wire and the die interfaces is modeled by Coulomb's coefficient $(\mu)$. Another important zone is the bearing cylindrical zone $(\mathrm{Hc})$, which accounts for calibrating the wire dimension.

During cold wiredrawing, there will always be internal stress of different signs and magnitude. Macroscopic residual stress is created by heterogeneous material flow. The stress distribution on cross and longitudinal sections in the wire must be in equilibrium. The macroscopic internal stress state in the drawn material is determined by metal forming conditions at each reducing pass ${ }^{5}$. The stress analyses of deforming distribution can be conducted to determine stress variation in the material during the wiredrawing process as the residual stress values in the drawn material vary ${ }^{6}$. These analyses are very important in the wiredrawing process optimization with multiple

*e-mail: martinez@demar.eel.usp.br 


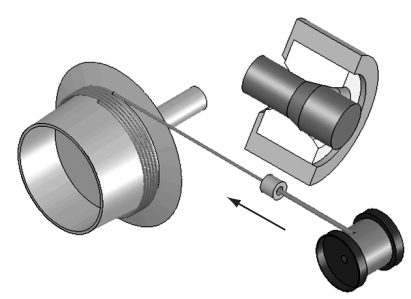

Figure 1. Scheme of the wiredrawing process.

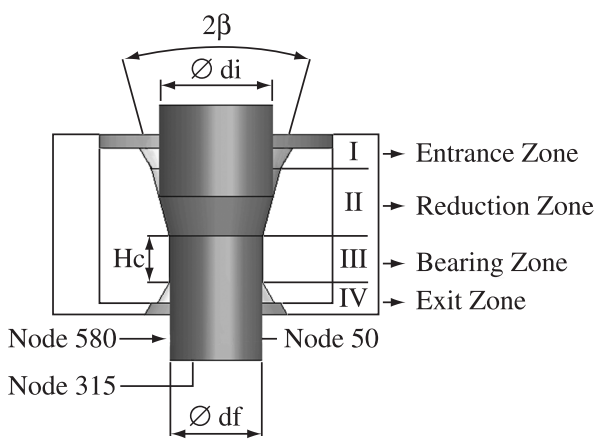

Figure 2. Scheme of internal die geometry.

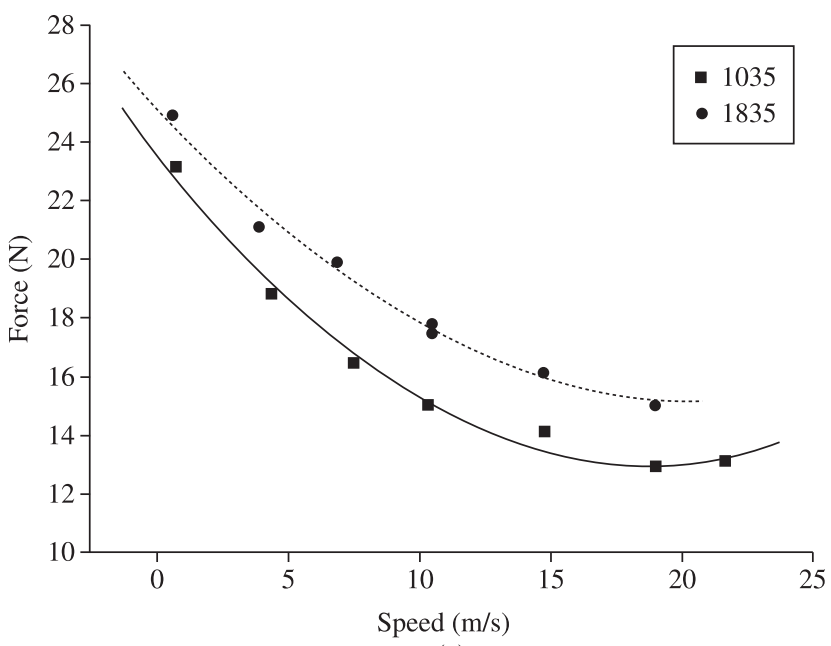

(a)

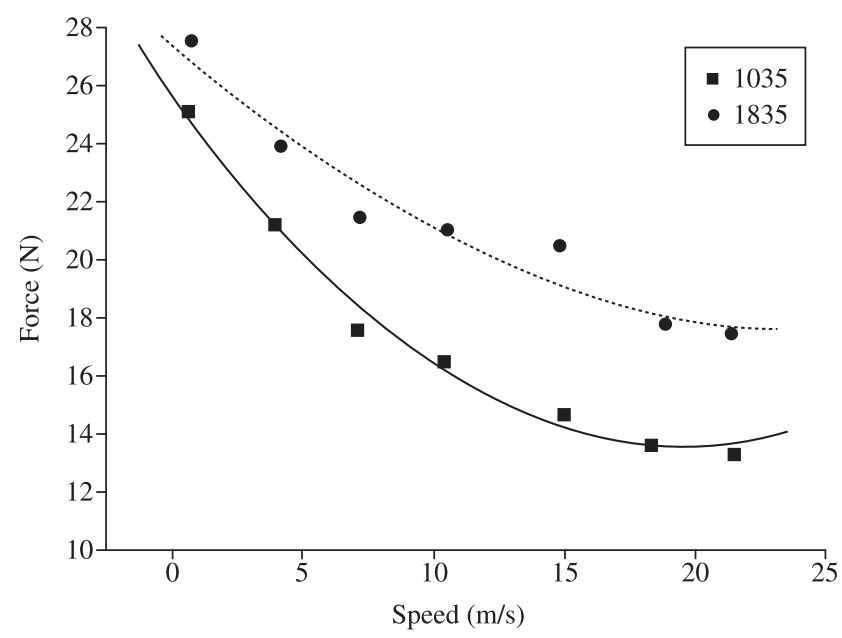

(c) dies, considering low possible contact forces and low values of internal and residual stresses, thus making the wiredrawing process more reliable.

This work presents results obtained in an experimental wiredrawing process, where the influence of the die geometry and the process parameters (velocity, wiredrawing force, and friction coefficient) in the radial and axial stress distributions on the deformation zone when $2 \beta=10^{\circ}$ and $18^{\circ}$ are studied.

The Finite Element Method (FEM) was used to determine the data variation and parameters research that cannot be experimentally obtained, and to provide numerical solutions, material flow curves, die stress and residual stress distributions in the drawn wire ${ }^{7}$.

\section{Materials and Methods}

Drawing of annealed electrolytic (ETP) copper wire, with $0.5 \mathrm{~mm}$ of original diameter, was carried out on a single die at velocities ranging from 1 to $23 \mathrm{~m} / \mathrm{s}$. A $19 \%$ reduction of the total cross section was used and the final diameter was $0.45 \mathrm{~mm}$, in one pass. Four sets of experimental material were produced using different geometry dies $(2 \beta+\mathrm{Hc})$. The water soluble commercial lubricant E-505/N was used in the experimental procedure. The friction coefficient $(\mu)$ was calculated by an upper limit method, by means of Equation 1 by

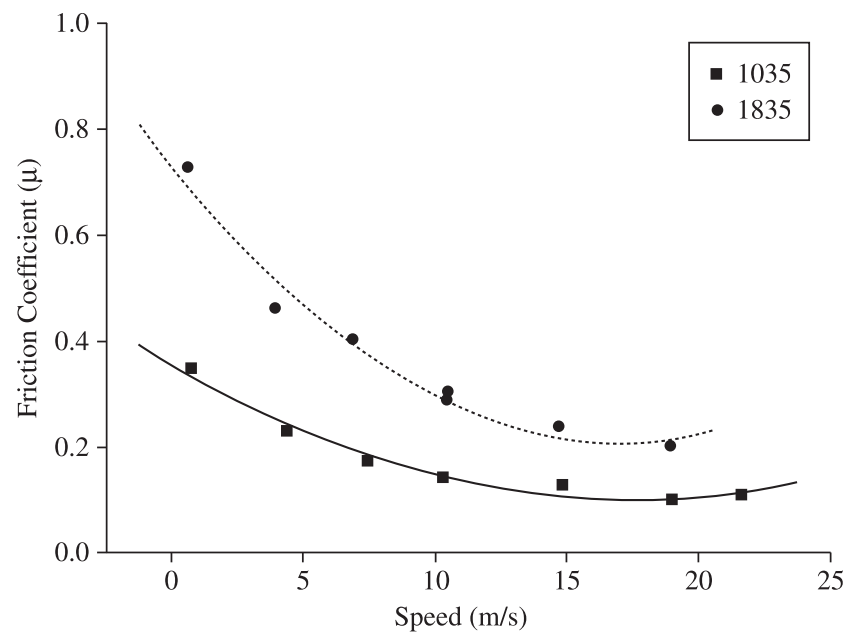

(b)

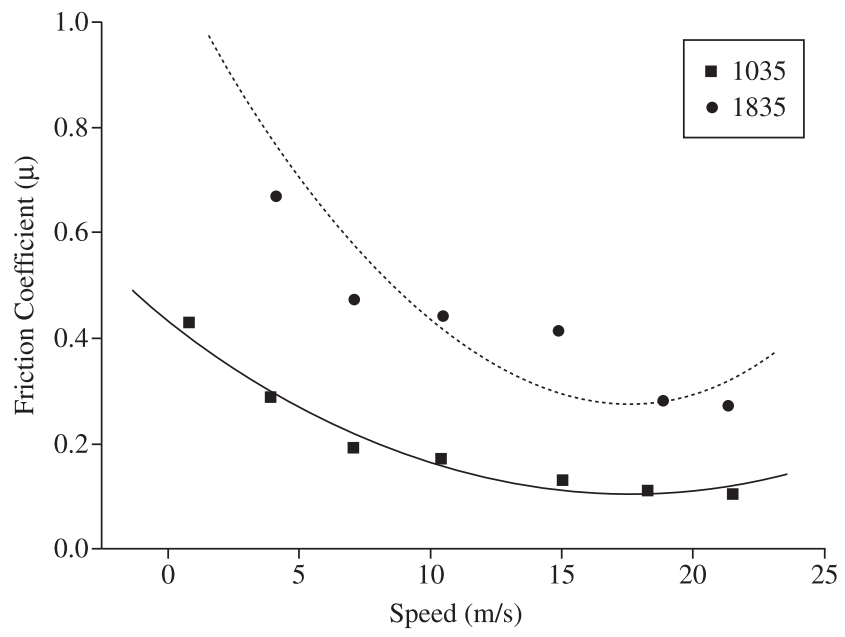

(d)

Figure 3. Wiredrawing forces and friction coefficient in relation to the wiredrawing velocity for $2 \beta=10^{\circ}$ and $2 \beta=18^{\circ}$, considering $\mathrm{Hc}=35 \%$ and $\mathrm{Hc}=50 \%$. 
Avitzur $^{8}$, in which the wiredrawing force values were used. A $0.5 \mathrm{~mm}$ diameter wire was used in obtaining the stress in Equation 1.

$$
\mu=\frac{\sigma_{0} \cdot\left(\frac{\sigma_{x b}}{\sigma_{0}}+2 \cdot f(\beta) \cdot \ln \frac{r_{i}}{r_{f}}+\frac{2}{\sqrt{3}} \cdot\left(\frac{\beta}{\operatorname{sen}^{2} \beta}-\cot g \beta\right)\right)-\sigma_{x f}}{2 \cdot\left(\left(\frac{H c}{r_{f}} \cdot \sigma_{x f}\right)-\left\{\sigma_{0} \cdot\left[(\cot \mathrm{g} \beta) \cdot\left(1-\frac{\sigma_{x b}}{\sigma_{0}}-\ln \frac{r_{i}}{r_{f}}\right) \cdot \ln \frac{r_{1}}{r_{f}}+\frac{H c}{r_{f}}\right]\right\}\right)}
$$

The dies used for the wiredrawing test (Figure 2) had $10^{\circ}$ and $18^{\circ}$ $2 \beta$ angles, and the lengths of Hc were $35 \%(1035,1835)$ and $50 \%$ $(1050,1850)$ of the wire final diameter $(0.45 \mathrm{~mm})$. The $2 \beta$ angles were maintained in the simulation for $\mathrm{Hc} 35 \%(1035,1835)$, for such configuration presented superior experimental results.

In this paper, the finite-element solver ${ }^{9} \mathrm{MARC}^{\circledR} 2001$ with graphical interface PATRAN ${ }^{\circledR} 2003$ r3 was used for the simulation of a wire drawing process to predict the distribution of residual stresses in drawn wires. Because of the axial symmetry in the drawing process, an axi-symmetric two-dimensional model was established to simulate the deformation. The workpiece consisted of 10 four-node-elements in the radial direction and 52 elements in the axial direction. The assumed hypotheses in the simulation are: i) the dies are rigid and the effect of friction between the die and the workpiece is modeled by Coulomb's coefficient $(\mu=0.1071)$, and ii) the wire is isotropic and non-linear elastic-plastic with an elastic modulus of $140 \mathrm{GPa}$ and a Poisson's ratio of 0.33 . The Von Mises yield criterion was used to describe the plastic behavior of the material.

\section{Results and Discussion}

Figures $3 \mathrm{a}$ and $3 \mathrm{c}$ show the results of the performed experiments and Figures $3 \mathrm{~b}$ and $3 \mathrm{~d}$ show the friction coefficients obtained through Avitzur (Equation 1).

Through the analysis of Figure 3 ( $b$ and d), the reduction of the wiredrawing force and the resulting reduction of the friction coefficient while increasing the speed were estimated. Such phenomena take place because the lubricant haulage increases at an enhanced velocity caused by the wedge effect between the deformation angle $(2 \beta)$ and the moving wire surface.

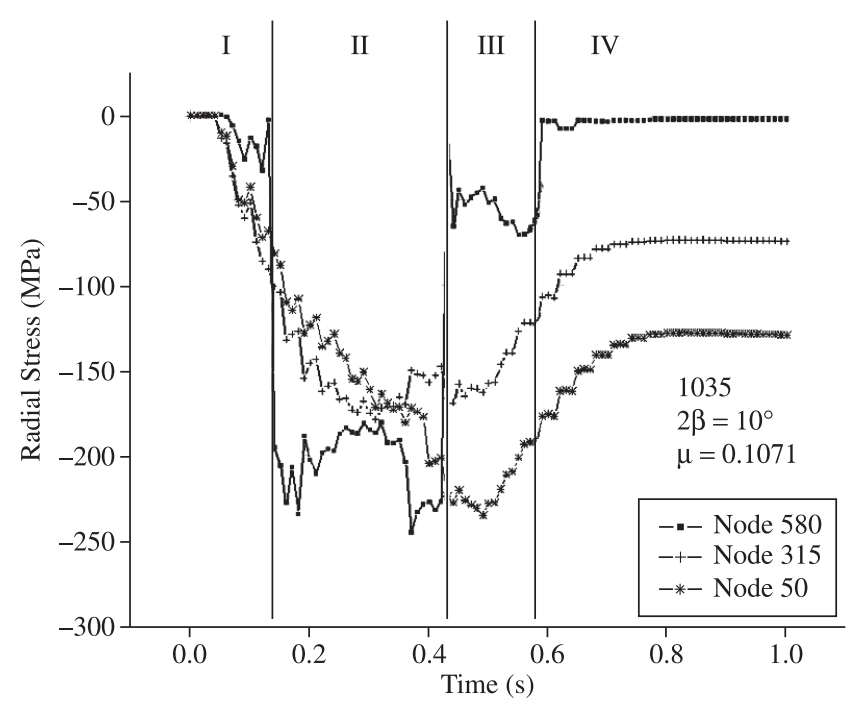

(a)
Figure 3 (a and c) show that the experiments in which $2 \beta$ is set at $10^{\circ}$ the wiredrawing force is lower within the whole range of velocities used in the experiments. The influence of the bearing cylindrical region $(\mathrm{Hc})$ on the wiredrawing force is made evident when $2 \beta$ is set at $18^{\circ}$ and $\mathrm{Hc}$ at 35 and $50 \%$ of the final diameter. The results show a decrease in the wiredrawing force for all speeds tested.

The radial and axial stress simulations (Figures 4 and 5 respectively) carried out in order to identify the best performing configuration. The simulations were performed in three distinct points across the wire while it passed through the die: i) surface region, (node 580); ii) intermediate region (node 315 ); and iii) central region (node 50), as shown in Figure 2.

The variations found in the entrance region (region I) show the existence of redundant work (Figure 4), which occurs due to the coming together of the wire and the deformation angle $(2 \beta)$. Redundant work appears at 2 distinct intensities when 2 different $2 \beta$ types are utilized, intensified when $2 \beta$ is increased from $10^{\circ}$ to $18^{\circ}$. In the deformation region (region II), radial compression increases for all nodes. The wire surface (node 580-zone II) is more compressed than other regions due to the wiredrawing die compression force on the outer layer of the wire. The influence of the deformation angle is apparent on the various layers of the wire. The trend is the lower the $2 \beta$ value, the lower the compression variation.

In the bearing region (region III), node 580 presents a change from compression stress to traction stress when $2 \beta=18^{\circ}$. The residual stress found in exit zone (region IV) shows its importance for the equilibrium of the compression and traction tensions. The simulation shows unevenness in the deformation, on several layers of the wire, caused by the wiredrawing die geometry. The stress values were much higher when the wiredrawing was performed dies with $2 \beta=10^{\circ}$. The results show the influence of the wiredrawing dies on the outer layers, and the consequent importance of lubrication in the reduction of friction coefficient between the wire and the die, for which the consequences are maintenance and the increase of die durability.

From the assessment of axial tensions in region I (Figure 5), redundant work was identified at a lower intensity when $2 \beta$ is set at a lower value, due to the enabling of the wire flowing. For the same reason, the tensions are more homogeneous in region II, even though nodes 315 and 50 present traction stress, and mixed tensions on node

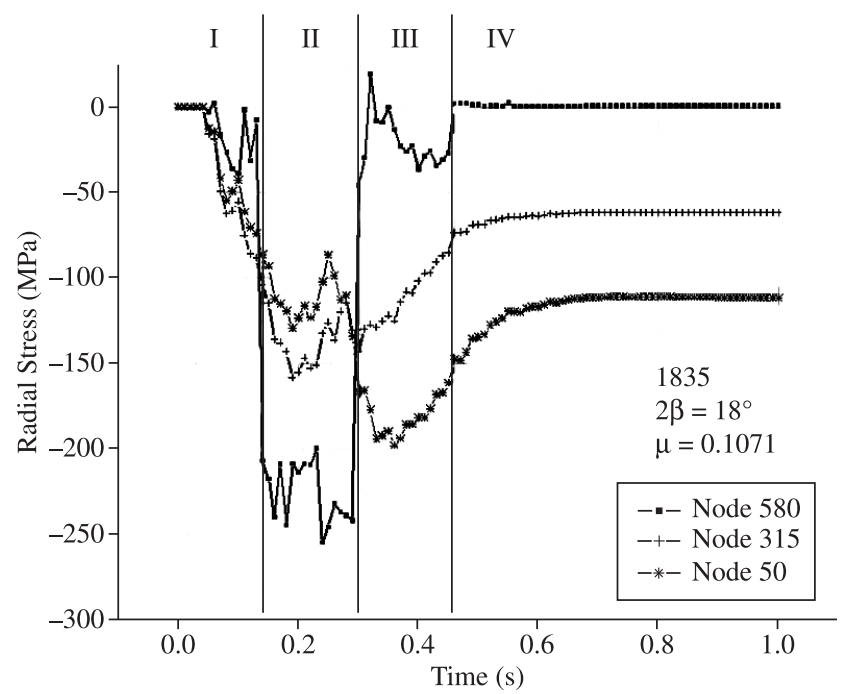

(b)

Figure 4. Radial Stress behavior during the wiredrawing process for a) $2 \beta=10^{\circ}$ and b) $2 \beta=18^{\circ}$. 


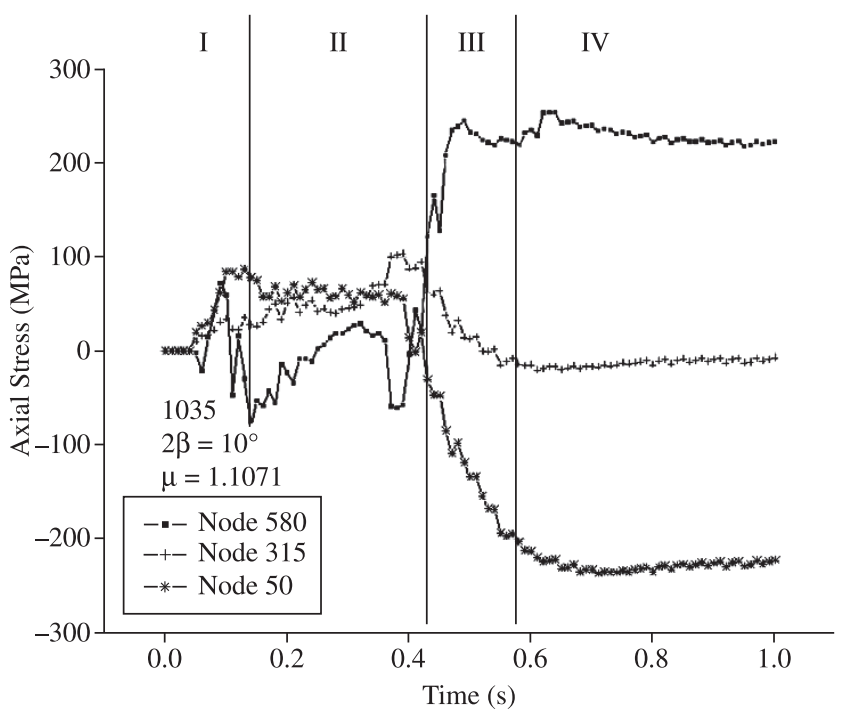

(a)

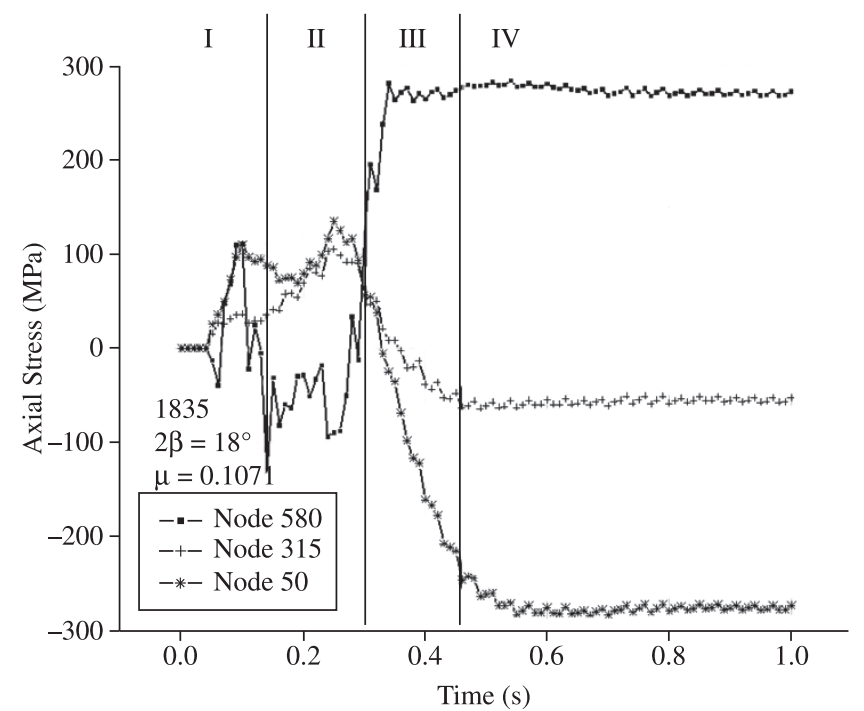

(b)

Figure 5. Axial Stress behavior during the wiredrawing process for a) $2 \beta=10^{\circ}$ and b) $2 \beta=18^{\circ}$.

580, where traction stress prevails. Characteristics such as the angle and the subsequent $2 \beta$ length are accountable for higher compression axial tensions when $2 \beta=18^{\circ}$.

Figures 5 (a) and (b) show the behavior of axial stress curves of the wiredrawing process. Although the wire does not suffer stress in the entrance zone (I), it presents tensile redundant work for all nodes. When the wire passes through the reduction zone (II), the surface (node 580) presents compressive stress while the intermediate and central regions (node 315 and 50) present tensile stress, mainly for $2 \beta=18^{\circ}$. In zone (III), this stress behavior changes on the surface and in internal regions (node 580 presents tensile stress while node 50 presents compression stress), and it is also more intense than that for $2 \beta=18^{\circ}$, showing the flow velocity contribution mainly in zone (III). The intermediate region (node 315 ) of zone (III) tends to get equilibrium for $2 \beta=10^{\circ}$, but it presents a little compression stress for $2 \beta=18^{\circ}$. The stress conditions of zone (IV) maintain it constant, i.e. maintaining the last values of axial stress in zone (III), for each node.

There is a connection between the tensile and compression stress in the wiredrawing process: the tensile stress is applied by the capstan machine and the compression stress is applied by the deformation zone of the die. Comparing Figures 4 and 5, the same level of radial and axial stress, around $250 \mathrm{MPa}$, showing a homogeneous stress state when $2 \beta=10^{\circ}$ was used, can be observed. The radial stress was around $250 \mathrm{MPa}$ while the axial stress was around $300 \mathrm{MPa}$ when $2 \beta=18^{\circ}$ was used, showing preponderance of tensile stress. In this case, the heterogeneous stress state, when $2 \beta=18^{\circ}$ was used, probably produces a wire with residual internal stress. In such case, more attention should be given to the application of the wire due to its mechanical properties.

\section{Conclusions}

- The wiredrawing force and the friction coefficient values decrease while the velocity increases.

- The die with $2 \beta=10^{\circ}$ presents wiredrawing force up to $23.2 \%$ lower and friction coefficient up to $60.5 \%$ lower than those for a die with $2 \beta=18^{\circ}$, considering $\mathrm{Hc}=35 \%$ and $\mathrm{Hc}=50 \%$.
- In dies with $2 \beta=10^{\circ}$, the wiredrawing force result is $4.4 \%$ lower and the friction coefficient value is $1.5 \%$ lower for $\mathrm{Hc}=35 \%$ than those obtained with dies with $\mathrm{Hc}=50 \%$.

- The Finite Element Method was applied in the simulation of wire drawing process to predict the distribution of residual stresses in drawn wires. The reduction zone (II) presents maximum radial stress on the surface and on the intermediate region, while the maximum axial stress is on the surface. Such behavior is more intense for $2 \beta=18^{\circ}$ than for $2 \beta=10^{\circ}$.

- The residual stress is permanent and thus determines important influence on fatigue properties, among other mechanical properties, after the wiredrawing process is completed. The total results of simulation show tensile axial stress applied on the surface of the wire, compression stress in the intermediate region, and intense residual stress level for $2 \beta=18^{\circ}$, showing the influence of stress on reduction zones.

\section{Acknowledgements}

Our appreciation to MSC Software of Brazil for providing PATRAN 2003 r3 and MARC 2001 software, and to FAPESP (Grants ${ }^{\circ} 15966-6$ and 02/00834-4)

\section{References}

1. Rubio EM, Camacho AM, Sevilha L and Sebastián MA. Calculation of the forward tension in drawing processes. Journal of Materials Processing Technology. 2005; 162-163 (2005) 551-557.

2. Camacho AM, Domingo R, Rubio EM and González C. Analysis of the influence of back-pull on drawing process by the Finite Element Method. Journal of Materials Processing Technology. 2005; 164-165 (2005): 1167-1174.

3. Shemenski RM. Wiredrawing by computer simulation. Wire Journal International. 1999; 32(4):166-183.

4. Nakagari A, Yamano T, Konaka M, Asakawa M, Sasaki W and Yoshida $\mathrm{K}$. Behavior of residual stress and drawing stress in conical-type die and circle-type die drawing by the FEM simulation and experiment. In: Proceedings of The Wire Association International Conference, Inc. Wire \& Cable Technical Symposium (WCTC), 69th Annual Convention; Atlanta, Geórgia, USA; May 1999 
5. Aernoudt E. Materials response to wiredrawing. Wire Journal International. 1989; 22(3): 53-75.

6. Doege E, Kröff A and Massai AM. Stress and strain analysis of automated multistage FEM-simulation of wiredrawing considering the backward force. Wire Journal International. 2000; 33(5):144-149.

7. Renz P, Steuff W and Kopp R. Possibilities of influencing residual stresses in drawn wires and bars. Wire Journal International. 1996; 29(1):64-69.

8. Avitzur B. Handbook of Metal Forming. New York, EUA: John Wiley and Sons; 1983.

9. PATRAN 2003 r3 software, MARC 2001 finite elements solver.

\section{Nomenclature:}

$\mathrm{di}=$ wire initial diameter, $(\mathrm{mm})$

$\mathrm{df}=$ wire final diameter, $(\mathrm{mm})$

$\mathrm{Hc}=$ bearing length, $(\mathrm{mm})$

$r_{i}=$ wire initial radius, $(\mathrm{mm})$

$\mathrm{r}_{\mathrm{f}}=$ wire final radius, $(\mathrm{mm})$

$\beta=$ reduction semi-angle, $(\mathrm{rad})$

$2 \beta=$ reduction angle, $(\mathrm{rad})$

$\mu=$ friction coefficient, (No unit)

$\sigma_{0}=$ yield stress, $(\mathrm{MPa})$

$\sigma_{\mathrm{xb}}=$ backward stress, $(\mathrm{MPa})$

$\sigma_{\mathrm{xf}}=$ wiredrawing stress, $(\mathrm{MPa})$

$\sigma_{\text {axial }}=$ axial stress, $(\mathrm{MPa})$

$\sigma_{\text {radial }}=$ radial stress, $(\mathrm{Mpa})$ 